\title{
Interference Analysis for Multi-Carrier Systems Over Fast-Fading Multipath Channels
}

\author{
Ahmad HAMDAN*†, Hussein HIJAZI ${ }^{\dagger}$, Laurent ROS*, Cyrille SICLET ${ }^{*}$, and Ali AL-GHOUWAYEL ${ }^{\ddagger}$ \\ ${ }^{*}$ Univ. Grenoble Alpes, CNRS, Grenoble INP ${ }^{1}$, GIPSA-Lab, 38000 Grenoble, France \\ ${ }^{\dagger}$ Lebanese International University, Department of Computer and Communications Engineering, Beirut, Lebanon \\ ${ }^{\ddagger}$ EFREI Paris, 94800 Villejuif, France
}

\begin{abstract}
In this paper, performance analysis for Multi-Carrier transmission using different pulse-shape-banks is provided for various scenarios. The pulse-shape-banks are selected based on different optimization criteria. These criteria fit into a range of considered scenarios requiring robustness against time dispersion or/and frequency dispersion while transmitting over a multi-path Rayleigh channel. Performance is provided in terms of interference power level versus normalized Doppler spread for various delay profiles while using a conventional correlator-based receiver. The interference localization properties of the pulse shapes and their importance are also discussed. When Doppler spread is negligible, it is shown that the Cyclic-Prefixed Orthogonal Frequency Division Multiplexing (CP-OFDM) has the best performance in terms of interference level. However, when the channel has a considerable variation, the Out of Band Energy (OBE) pulse shape appears to have better performance. For extreme-case scenarios, where the channel is significantly affected by both delay and Doppler spreads, we recommended to consider the interference localization properties of the pulse shapes instead of interference level.

Index Terms-Rayleigh Channel, Doppler, Fast Fading, MultiCarrier, OFDM, CP-OFDM, FBMC.
\end{abstract}

\section{INTRODUCTION}

$\mathbf{H}$ IGH symbol rate transmission has been in research for decades due to the continuously increasing demand for high data rates. Achieving higher data rates is challenging especially in environments suffering from tough impairments like mobile communication channels. Such mobile wireless channels suffer from multi-path propagation. When the multipath propagation delay spread is not negligible compared to the symbol duration, transmitting with a high symbol rate over a single-carrier system, which is expected to have a short symbol duration, makes the system severely affected by Inter-Symbol Interference (ISI) and frequency selectivity. To overcome this weakness, Multi-Carrier (MC) waveforms were introduced. MC waveforms are based on transmitting the data over multiple carriers, called sub-carriers. This is done by splitting the data stream into multiple sub-streams with lower data rates. Each stream is transmitted over a subcarrier with narrower bandwidth. By performing such datacarrier multiplexing, symbol duration is expanded making the transmission more resilient to delay spreads. Classically, Orthogonal Frequency Division Multiplexing (OFDM) is used to perform this multiplexing due to its various benefits:

This work has been partially supported by the LabEx PERSYVAL-Lab (ANR11-LABX-0025-01) funded by the French program Investissement d'avenir.

${ }^{1}$ Institute of Engineering Univ. Grenoble Alpe. simplicity of implementation [1], multiple-access capabilities [2] and low complexity equalization [3] thanks to Cyclic-Prefix (CP) in addition to ISI-free communication if the delay spread is shorter than the $\mathrm{CP}$ duration. However, another challenge is exhibited by the mobile communication channels due to the possible displacement of the receiver with respect to the transmitter during transmission, which will create variation in the attenuation and phase shift caused by the channel due to the Doppler effect. Such phenomenon has more impact on waveforms with longer symbol duration as they will include more variations. This makes the MC transmissions less resistant to channel's variations and can be significantly affected by the exhibited Doppler spread introducing Inter-Carrier Interference (ICI). Requirements of mobile communications applications might vary between classical MC systems requiring resistance to multi-path propagation, waveforms that require short Transmission Time Interval (TTI) -as in Ultra-Reliable Low-Latency Communications (URLLC) [4] (making them more vulnerable to multi-path environments), and systems requiring resilience to both time and frequency dispersion as in Vehicle-to-Vehicle (V2V) communications [5]. To allow the applicability of the requirements on MC systems, FilterBank Multi-Carrier (FBMC) [6] and Bi-orthogonal Frequency Division Multiplexing (BFDM) [7] are used. FBMC uses pulse shapes different than the Rectangular (Rect) pulse adopted in OFDM, and BFDM allows the use of a pulse shape at the receiver different from, but bi-orthogonal to, the pulse shape used at the transmitter. The configuration of the pulse shape defines major properties of the communication system in terms of resistance to channel's impairments. Therefore, it is required to provide a comparison between different pulse shapes originally designed, each on specific criterion, to show robustness against doubly dispersive channels. Up to the authors' knowledge, such comprehensive analysis is missing from the literature. The contribution in this paper to provide this analysis is through:

- providing a generalized system model that is valid for multi-carrier communication over doubly-dispersive channels and deriving the theoretical expected received power at each time-frequency slot,

- analyzing the generalized multi-carrier system's performance in doubly-dispersive channels using the derived theoretical values of ISI and ICI versus normalized 


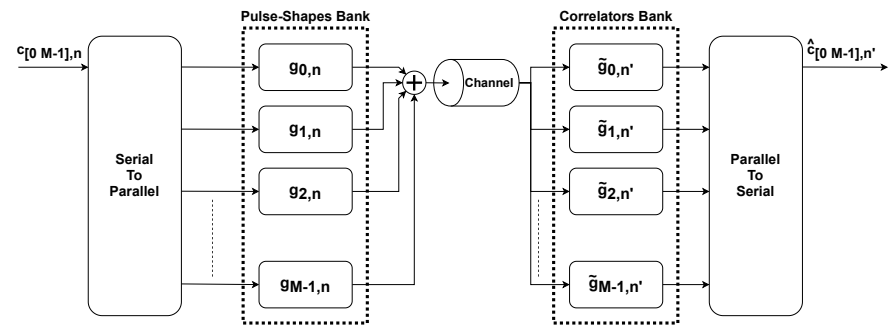

Fig. 1. Multi-Carrier System Schematic.

Doppler spread assuming Rayleigh channel with Jakes' Doppler spectrum (Clarke model [8], [9]). This is done for pulse shapes using different optimization criteria,

- analyzing the time-frequency localization of interference (ISI and ICI) by using the derived theoretical expression versus the offset relative to the index of the desired timefrequency slot,

- and providing Monte-Carlo simulation results that validate the provided theoretical interference formulas.

The rest of this paper is organized as follows: section II describes the system model, while section III discusses the pulse shapes being used, the criteria they are selected on, and the channel delay profiles taken into account. Simulation results are provided and discussed in section IV. Finally, the paper is concluded in section V. The details of the theoretical derivation of the expected interference power are provided in the appendix.

\section{SYSTEM MODEL}

The MC system used in this paper reflects the schematic shown in Fig. 1. The aim is to minimize the cross-talk between sub-carriers and consecutive symbols by trying to satisfy the relation:

$$
\left\langle\boldsymbol{g}_{m, n}, \tilde{\boldsymbol{g}}_{m^{\prime}, n^{\prime}}\right\rangle \approx \begin{cases}\text { Const, } & \text { if } m=m^{\prime} \text { and } n=n^{\prime} \\ 0 & \text { otherwise, }\end{cases}
$$

where:

- $\langle\boldsymbol{x}, \boldsymbol{y}\rangle$ is the inner product between $\boldsymbol{x}$ and $\boldsymbol{y}$ such that $\langle\boldsymbol{x}, \boldsymbol{y}\rangle=\sum_{q} x[q] y^{*}[q]$.

- $\boldsymbol{g}_{m, n}$ is the transmitting pulse shape of the $m^{t h}$ sub-carrier of the $n^{t h}$ transmitted MC symbol.

- $\tilde{\boldsymbol{g}}_{m^{\prime}, n^{\prime}}$ is the receiving pulse shape used to recover (by correlation) the $m^{\prime t h}$ sub-carrier of the $n^{\prime t h}$ received MC symbol. In the case of conventional matched filter receiver, $\tilde{\boldsymbol{g}}_{m^{\prime}, n^{\prime}}=\boldsymbol{g}_{m^{\prime}, n^{\prime}}$. This type of receivers is used in this work except for the Rect pulses using the CP-OFDM implementation.

Let $M$ be the number of sub-carriers, $N$ be the interval - in samples - between two consecutive MC symbols such that for a sample duration $T_{S a}$ the MC symbol duration (interval) is $T_{S}=N T_{S a}, K$ be the time span of a pulse shape in terms of MC symbols (number of pulse-shape samples $/ N$ ) such that $K N$ is an integer, and (equivalently) $K-1$ be the overlapping factor. As the pulse shapes have a defined length of $K N$, the prototype transmitting pulse shape $g[k]$ and the prototype receiving pulse shape $\tilde{g}[k]$ are supported for $k \in[0 ; K N-1]$, and $g[k]=\tilde{g}[k]=0$ otherwise. The $q^{t h}$ sample transmitted due to an input symbol $c_{m, n}$ on the $m^{t h}$ sub-carrier of the $n^{t h}$ $\mathrm{MC}$ symbol is written as:

$$
s_{m, n}[q]=c_{m, n} g_{m, n}[q] .
$$

Summing over all the sub-carriers, the $q^{t h}$ sample of $n^{\text {th }}$ MC symbol for the input symbols $c_{m, n}$ will be:

$$
\begin{aligned}
s_{n}[q] & =\sum_{m} s_{m, n}[q] \\
& =\sum_{m} c_{m, n} g_{m, n}[q],
\end{aligned}
$$

such that the $q^{t h}$ sample of the transmitting pulse shape $\boldsymbol{g}_{m, n}$ is:

$$
g_{m, n}[q]=g[q-n N] e^{j 2 \pi \frac{m(q-n N)}{M}} .
$$

Using Eq. 3 and Eq. 4 the sample stream of the all transmitted symbols summed over $n$ and $m$ will be:

$$
s[q]=\sum_{n} \sum_{m} c_{m, n} g[q-n N] e^{j 2 \pi \frac{m(q-n N)}{M}} .
$$

Since the aim of the study is to measure the inner robustness of the waveforms, the noise will be ignored. Thus, the signal at the input of the receiver will be expressed as:

$$
r[q]=(h * s)[q]=\sum_{l=0}^{L-1} h[q, l] s[q-l],
$$

such that:

- $r[q]$ is the $q^{t h}$ sample of received MC symbol stream $\boldsymbol{r}$,

- $h[q, l]$ is the $q^{\text {th }}$ sample of the $l^{\text {th }}$ discrete time-varying Wide Sense Stationary Uncorrelated Scatterers (WSSUS) channel [10],

- $L$ is the number of paths of the discrete-time channel,

- and $*$ is the discrete convolution operator.

Using operations matched to those at the transmitter, the received constellation symbol at the $m^{\prime t h}$ sub-carrier and the $n^{\prime t h}$ MC symbol $c_{m^{\prime}, n^{\prime}}$ can be expressed as:

$$
\hat{c}_{m^{\prime}, n^{\prime}}=\left\langle\boldsymbol{r}, \tilde{\boldsymbol{g}}_{m^{\prime}, n^{\prime}}\right\rangle=\sum_{q} \tilde{g}_{m^{\prime}, n^{\prime}}^{*}[q] r[q],
$$

having the $q^{\text {th }}$ sample of the receiving pulse (or Hermitian symmetric of the receiving filter's impulse response) $\tilde{\boldsymbol{g}}_{m^{\prime}, n^{\prime}}$ as:

$$
\tilde{g}_{m^{\prime}, n^{\prime}}[q]=\tilde{g}\left[q-n^{\prime} N\right] e^{j 2 \pi \frac{m^{\prime}\left(q-n^{\prime} N\right)}{M}} .
$$

Using Eq. 7 and Eq. 8:

$$
\begin{aligned}
\hat{c}_{m^{\prime}, n^{\prime}}= & \left\langle\boldsymbol{r}, \tilde{\boldsymbol{g}}_{m^{\prime}, n^{\prime}}\right\rangle \\
= & \sum_{q} \tilde{g}^{*}\left[q-n^{\prime} N\right] r[q] e^{-j 2 \pi \frac{m^{\prime}\left(q-n^{\prime} N\right)}{M}} \\
= & \sum_{l} \sum_{q} \sum_{n} \sum_{m} \tilde{g}^{*}\left[q-n^{\prime} N\right] \\
& \times h[q, l] g[q-n N-l] c_{m, n} \\
& \times e^{-j 2 \pi \frac{(q-n N)\left(m^{\prime}-m\right)-m^{\prime} N\left(n^{\prime}-n\right)+l m}{M}},
\end{aligned}
$$


and for a specific sub-carrier $m$ and symbol $n$ :

$$
\begin{aligned}
\left.\hat{c}_{m^{\prime}, n^{\prime}}\right|_{m, n}= & \sum_{l} \sum_{q} \tilde{g}^{*}\left[q-n^{\prime} N\right] \\
& \times h[q, l] g[q-n N-l] c_{m, n} \\
& \times e^{-j 2 \pi \frac{(q-n N)\left(m^{\prime}-m\right)-m^{\prime} N\left(n^{\prime}-n\right)+l m}{M}} .
\end{aligned}
$$

The system model just defined is depicted in Fig. 1 such that the design of $g_{m, n}$ and $\tilde{g}_{m^{\prime}, n^{\prime}}$ aims to satisfy the relation in Eq. 1. Accordingly, the power received due to the transmission of subcarrier $m$ of symbol $n$ received at subcarrier $m^{\prime}$ of symbol $n^{\prime}$ can be obtained by:

$$
P\left(m, m^{\prime}, n, n^{\prime}\right)=\left(\left.\hat{c}_{m^{\prime}, n^{\prime}}\right|_{m, n}\right)\left(\left.\hat{c}_{m^{\prime}, n^{\prime}}^{*}\right|_{m, n}\right) .
$$

Assuming WSSUS Jakes' model [8] Rayleigh channel, with independent and identically distributed (i.i.d) input symbols and unitary average power $\left(E\left[c_{m, n} c_{m, n}^{*}\right]=1\right)$, the expectation of the received power is (derivation in Appendix A):

$$
\begin{aligned}
\bar{P}_{\Delta}=\sum_{l} \sum_{\gamma} \sum_{\gamma^{\prime}} g\left[\gamma+N \Delta_{S}-l\right] g^{*}\left[\gamma^{\prime}+N \Delta_{S}-l\right] \\
\times \tilde{g}^{*}[\gamma] \tilde{g}\left[\gamma^{\prime}\right] e^{j 2 \pi \frac{\Delta_{C}\left(\gamma^{\prime}-\gamma\right)}{M}} \\
\times \sigma_{l}^{2} J_{0}\left(2 \pi F_{d} T_{S a}\left(\gamma^{\prime}-\gamma\right)\right),
\end{aligned}
$$

where:

- $J_{0}$ is the Bessel function of the first kind and order 0,

- $\sigma_{l}^{2}$ the power/variance of the $l^{t h}$ path,

- $F_{d}$ is the maximum Doppler frequency,

- and the index $\Delta=\left[\Delta_{S}, \Delta_{C}\right]$ such that $\Delta_{S}=n^{\prime}-n$ and $\Delta_{C}=m^{\prime}-m$.

$\bar{P}_{\Delta}$ can be used to compute the ISI components for $\Delta_{S} \in$ $\mathbb{Z}^{*} \& \Delta_{C} \in \mathbb{Z}$, and the ICI components for $\Delta_{S}=0 \& \Delta_{C} \in$ $\mathbb{Z}^{*}$. In the next section, the pulse shapes and the channel conditions being considered for simulation are discussed.

\section{Considered Pulse Shapes and Channels}

As discussed before and shown in Eq. 12, the interference level of the MC system is affected by two factors: 1) pulse shapes, which tries to match the orthogonality/bi-orthogonality condition expressed in Eq. 1,2) the channel's properties, which are represented in Eq. 12 through the power of the paths $\sigma_{l}^{2}$ reflecting the delay profile, and through the normalized Doppler spread $F_{d} T_{S}=N F_{d} T_{S a}$ reflecting the channel's variation. Therefore, in this section, we will discuss the pulse shapes and the channel setups used for comparison. As mentioned in section I, MC transmission is used for various scenarios, consequently, different pulse shape optimization criteria should be used based on the scenario being considered. Therefore, in this paper, the following pulse shapes are taken into consideration for the comparative study:

- Rectangular (Rect) pulse shape emphasizing the CPOFDM implementation by having a $N T_{S a}$ Rect pulse at the transmitter and a shorter zero-leaded $M T_{S a}$ Rect pulse correlator at the receiver. The leading transmitted $N-M$ samples at the transmitter represent the $\mathrm{CP}$ which is removed at the receiver by the leading zeros multiplication.
TABLE I

SP, EPA, EVA, AND M-ETU CHANNELS RESAMPLED TO THE DISCRETE-TIME CHANNEL WITH SUB-CARRIER SPACING $30 \mathrm{kHz}$ AND $N=36$

\begin{tabular}{|c|c|c|c|c|}
\hline \multirow{2}{*}{ Tap index $l$} & \multicolumn{4}{|c|}{ Power at delay $l \sigma_{l}^{2}$} \\
\cline { 2 - 5 } & SP & EPA & EVA & M-ETU \\
\hline 0 & $1.0 \mathrm{e}+00$ & $8.3 \mathrm{e}-01$ & $6.9 \mathrm{e}-01$ & $6.1 \mathrm{e}-01$ \\
1 & & $1.6 \mathrm{e}-01$ & $2.9 \mathrm{e}-01$ & $1.8 \mathrm{e}-02$ \\
2 & & $5.6 \mathrm{e}-03$ & $1.6 \mathrm{e}-02$ & $2.6 \mathrm{e}-03$ \\
3 & & $1.6 \mathrm{e}-05$ & $2.9 \mathrm{e}-03$ & $2.5 \mathrm{e}-02$ \\
4 & & & $4.4 \mathrm{e}-05$ & $2.6 \mathrm{e}-01$ \\
5 & & & $1.1 \mathrm{e}-06$ & $7.6 \mathrm{e}-02$ \\
6 & & & & $4.7 \mathrm{e}-03$ \\
7 & & & & $1.7 \mathrm{e}-04$ \\
8 & & & & $4.7 \mathrm{e}-07$ \\
\hline
\end{tabular}

- Out of Band Energy (OBE) pulse shape [11]: optimized to minimize the out of band $\left[0 ; F_{S}\right]$ energy for sub-carrier spacing $F_{S}$ under the constraint of limiting the pulse shape to one symbol duration ( $K=1$ - non-overlapping) and maintaining orthogonality to similar pulses centered at frequencies $k F_{S}$ away from the pulse's center where $k \in \mathbb{Z}$.

- Time Frequency Localized (TFL) pulse shape [11]: optimized to maximize the time-frequency localization under the constraint of limiting the pulse shape to one symbol duration ( $K=1$ - non-overlapping) and maintaining orthogonality to similar pulses centered at frequencies $k F_{S}$ away from the pulse's center where $k \in \mathbb{Z}$.

- Root Raised Cosine (RRC) pulse shape [12]: optimized to limit the frequency bandwidth of the pulse and maintaining orthogonality to similar pulses centered at time $k T_{S}$ away from the pulse's center where $k \in \mathbb{Z}$. The notation $\mathrm{RRC}^{\alpha}$ is used for an RRC pulse with a roll-off factor $\alpha$. $\mathrm{RRC}^{\alpha}$ pulses maintain orthogonality to other sub-carriers when $\alpha>\frac{N}{M}-1$.

- Gaussian (Gauss) pulse shape: optimized to maximize the time-frequency localization relaxing the constraint of limiting the pulse shape duration to one symbol interval ( $K>1-$ overlapping). It necessarily introduces interference since the Gauss pulses are not orthogonal.

The delay profiles considered in this paper, shown in Table I, are: Single Path Channel (SP), Extended Pedestrian A model (EPA), Extended Vehicular A model (EVA), and Modified Extended Typical Urban model (M-ETU) channels from [13] resampled to the discrete time channel with sub-carrier spacing $F_{S}=30 \mathrm{kHz}$ and $N=36$. Beside their impact on the channels' taps resampling, these parameters define other system parameters like the total bandwidth of the system $B=M F_{S}$, the sample time $T_{S a}=1 / B=1 / M F_{S}$, and the symbol time interval $T_{S}=N T_{S a}=N / M F_{S}$. The next section provides the analysis of MC transmission - using the pulse shapes mentioned - over channels with the listed delay profiles for several Doppler spread values. 


\section{Results AND AnAlysis}

In this section the Doppler Spread will not be pre-defined as the interference level versus normalized Doppler spread is studied. The delay profiles scenarios provided in Table I will be used for the simulation. The following setup is used: the number of sub-carriers $M=32$, the number of samples spacing between two consecutive symbols $N=36$, the size of the pulse shape (in terms of $N$ ) $K=15$, and the receiver and transmitter pulses are matched (except for Rect pulse using CP-OFDM implementation). Solid line plots are obtained by Eq. 12, while the markers reflect 5000-observation MonteCarlo simulations. A large value of $K$ is selected to limit the truncation effects on the RRC pulse shape which does not affect time-limited pulses (Rect, OBE, and TFL) as they have a zero value for $q \notin[0 ; N-1]$. The Gauss pulse shape is configured to have equal spreading, in frequency normalized to sub-carrier spacing, and in time normalized to symbol time. This configuration of Gauss pulse shape is negligibly affected by truncation for $K \geq 3$. For the $\mathrm{RRC}^{\alpha}$ pulses, two values of the roll-off $\alpha$ are considered for this work:

- $\alpha=\frac{1}{8}$ : restricted to having ICI-free communication for static channels along with the preset values of $M$ and $N$, the largest possible $\alpha$ is $\alpha=\frac{N}{M}-1=\frac{1}{8}$.

- $\alpha=1$ : provides the minimal time-domain support (or equivalently the best time localization) of an RRC pulse, yet loses orthogonality to neighbor sub-carriers due to having $\alpha>\frac{N}{M}-1$

The interference level in this section is calculated in terms of normalized Inter-Symbol and Inter-Carrier Interference (ISCI):

$$
\text { ISCI }=\text { ISI }+ \text { ICI }=\sum_{\Delta \neq[0,0]} \frac{\bar{P}_{\Delta}}{\bar{P}_{[0,0]}} .
$$

Fig. 2 provides comparisons for the single path scenario for a) ISCI versus $F_{d} T_{S}$, b) received power versus symbol offset $\Delta_{S}$, and c) received power versus sub-carrier offset $\Delta_{C}$. In the latter two sub-figures, we have set $F_{d} T_{S}=0.2$. Three scenarios can be extracted from Fig. 2-a):

Scenario 1) Single path channels with negligible Doppler spread (i.e. $F_{d} \cdot T_{s}<0.01$ ). This scenario is not sensitive to neither Doppler spread issues, nor delay spread issues. In such a scenario, it is shown that the time-limited pulses (Rect, OBE, TFL, ...) are ISI-free. It also appears how the $\mathrm{RRC}^{\frac{1}{8}}$ introduces interference for static channels due to time-truncation. It is clear how $\mathrm{RRC}^{1}$ and Gauss have high interference for static channels since they are non-orthogonal pulses for this multicarrier setup.

Scenario 2) Single path channels with slightly higher Doppler spread (around $F_{d} T_{S}=0.04$ ). At this level, OBE has the best performance among time-limited pulses as it is optimized to have minimum frequency-domain spreading. $\mathrm{RRC}^{\frac{1}{8}}$ provides the best performance in terms of interference level. This good performance is obtained thanks to the optimization of the RRC pulse to have minimum bandwidth, which allows to minimize the effect of the Doppler spread in frequency domain. However,
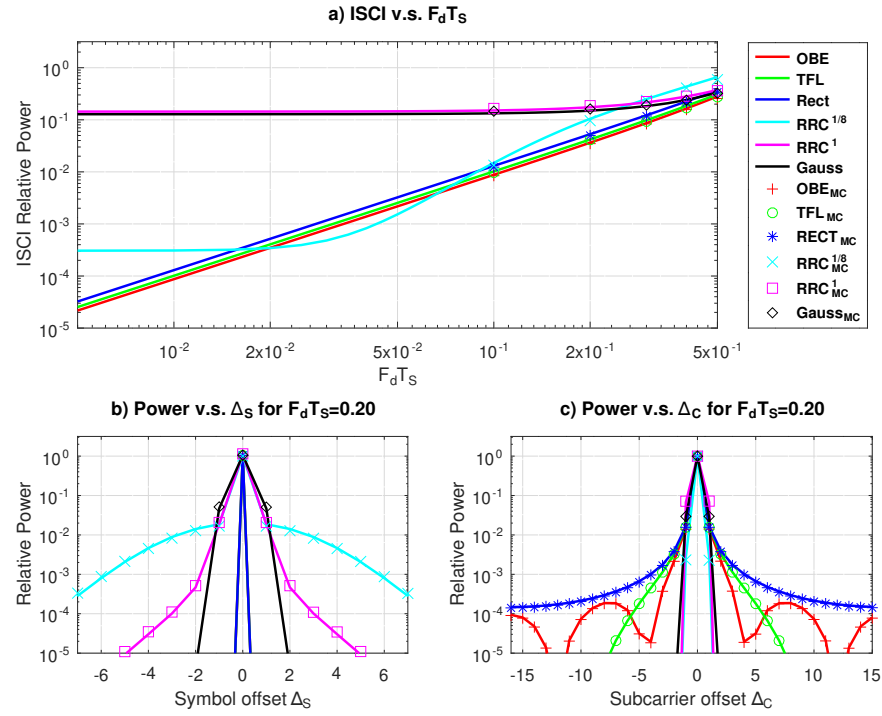

Fig. 2. Comparison for the SP scenarios for a) ISCI versus $F_{d} T_{S}$, b) received power versus symbol offset $\Delta_{S}$, and c) received power versus sub-carrier offset $\Delta_{C}$. b) and c) for $F_{d} T_{S}=0.2$.

this is not true for higher Doppler spread as will be discussed in the next scenario.

Scenario 3) Fast varying single path channels with normalized Doppler spread $>0.1$. It could be expected to get the best performance with $\mathrm{RRC}^{\frac{1}{8}}$ by assuming that the main concern for Doppler spread is ICI, but that is not the case. It is shown that for fast-varying channels, $\mathrm{RRC}^{\frac{1}{8}}$ has a significant increase in the interference level, where this interference is actually significantly including Doppler-generated-ISI and not limited to Doppler-generated-ICI. It appears that the channel variation causes the $\operatorname{RRC}^{\frac{1}{8}}$ pulse to have its orthogonality, to the pulses at other time slots, broken, making it the pulse that is affected the most by the interference increase when the normalized Doppler spread increases. It is also shown how OBE has a performance slightly better than Rect and TFL as it is designed to have minimum frequency domain spreading.

Fig. 2-b) and c) show another interference-related property, which is interference spreading/localization, or the power received at every time-frequency slot due to the transmission at a specific time-frequency slot. These figures illustrate how the power of the time-limited pulses is spread over all the spectrum in the frequency domain while introducing no ISI for single path channels. On the other hand, the frequency-limited pulses have localized ICI but significantly spreading ISI. The compromise between the two cases, although having the highest interference levels, are the $\mathrm{RRC}^{1}$ and Gauss pulse shapes which have the most localized interference. This property can be beneficial at various levels like interference cancellation techniques, and waveform density reduction in extreme cases to reduce the interference levels to an acceptable range.

Figs. 3-5 provide comparisons for EPA, EVA, and M-ETU channel scenarios respectively for a) ISCI versus $F_{d} T_{S}$, b) received power versus symbol offset $\Delta_{S}$, and c) received power versus sub-carrier offset $\Delta_{C}$. In the latter two sub-figures, we 
a) ISCl v.s. $F_{d} T_{S}$

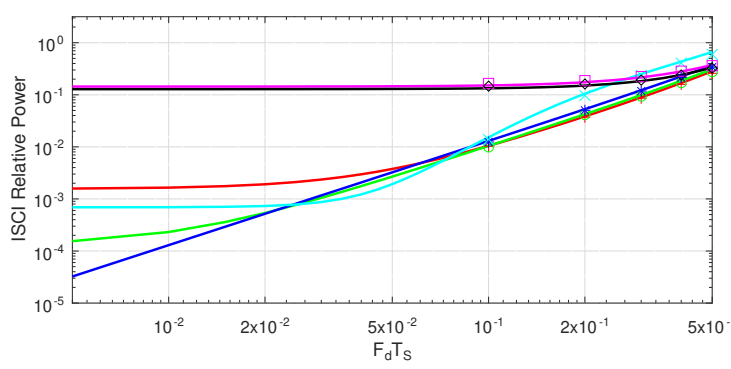

b) Power v.s. $\Delta_{S}$ for $F_{d} T_{S}=0.20$
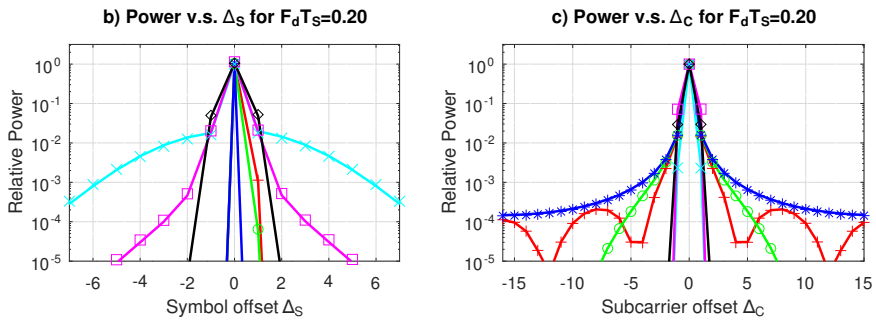

Fig. 3. Comparison for EPA channel scenarios for a) ISCI versus $F_{d} T_{S}$, b) received power versus symbol offset $\Delta_{S}$, and c) received power versus sub-carrier offset $\Delta_{C}$. b) and c) for $F_{d} T_{S}=0.2$.
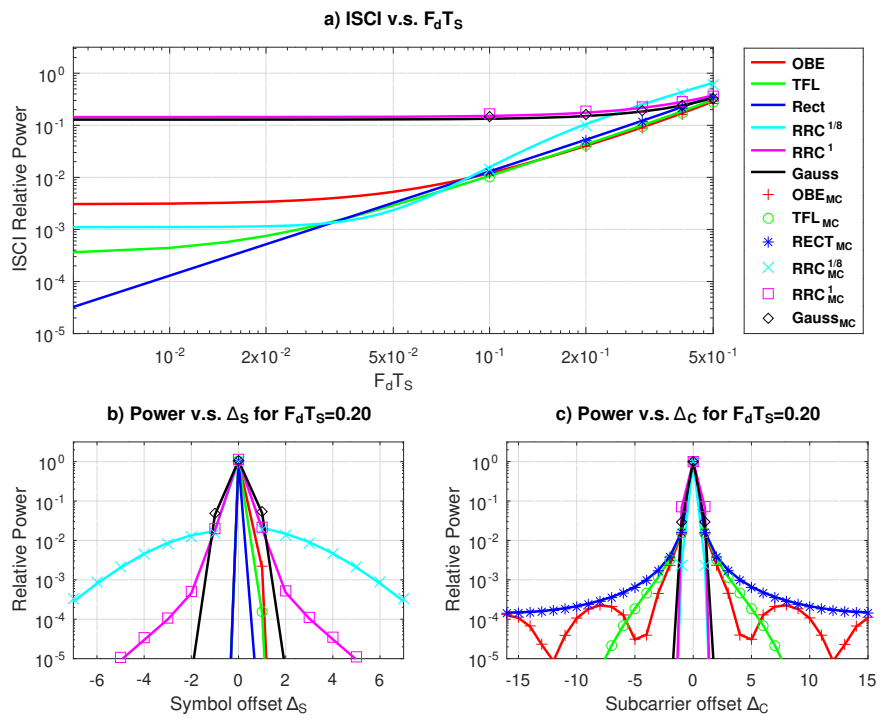

Fig. 4. Comparison for EVA channel scenarios for a) ISCI versus $F_{d} T_{S}$, b) received power versus symbol offset $\Delta_{S}$, and c) received power versus sub-carrier offset $\Delta_{C}$. b) and c) for $F_{d} T_{S}=0.2$.

have set $F_{d} T_{S}=0.2$. The results in these figures are quite similar to those in Fig. 2 with some distinctions to be mentioned below:

1) An increase in causal ISI is observed for all the pulses especially for $\Delta_{S}=1$, and the interference level increases as the delay spread increases.

2) TFL and OBE pulses introduced causal ISI (as they are tightly aligned in the time domain) due to multi-path propagation even for negligible Doppler spread, with TFL better for low Doppler spreads as it is optimized to have maximum time-frequency localization.

3) ICI is increased (except for Rect with $\mathrm{CP}>$ maximum
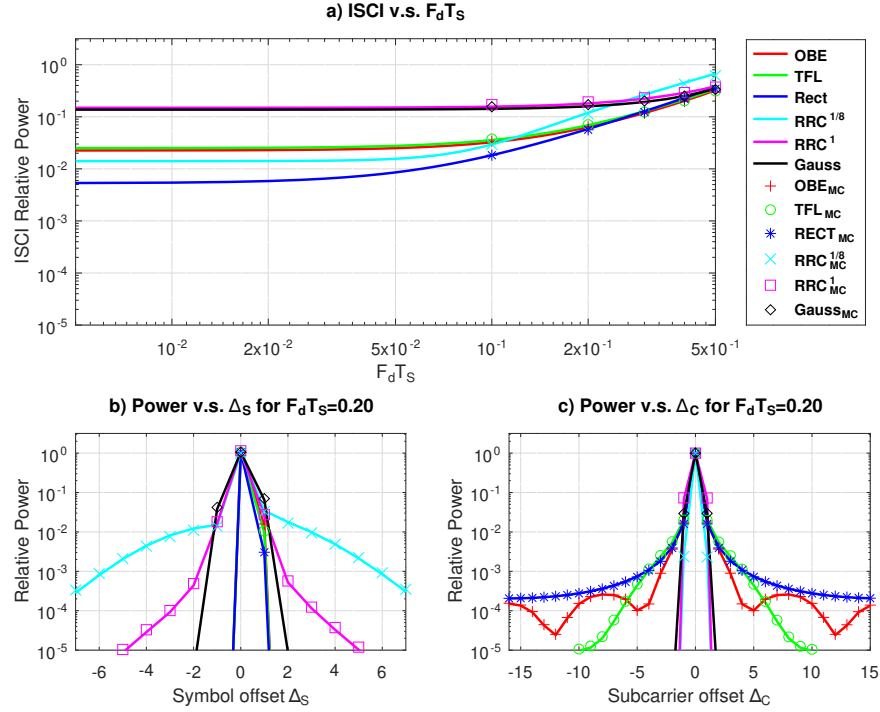

Fig. 5. Comparison for M-ETU channel scenarios for a) ISCI versus $F_{d} T_{S}$, b) received power versus symbol offset $\Delta_{S}$, and c) received power versus sub-carrier offset $\Delta_{C}$. b) and c) for $F_{d} T_{S}=0.2$.

delay) due to having the delayed versions of the transmitted pulse not orthogonal to the receiving pulse for other sub-carriers.

4) Rect pulse maintains, approximately, orthogonality for low Doppler spread as far as the delay spread is bounded to the CP duration as in Fig. 3 and Fig. 4. However, it is affected when the delay spread exceeds the $\mathrm{CP}$ duration as shown in Fig. 5, an effect similar to the case of TFL and OBE pulses.

5) For all scenarios, Gauss and $\mathrm{RRC}^{1}$ are the least affected by spreading impairments and maintain the best localization, but generating interference even in ideal scenarios. It was shown in our previous work [14] how the interference localization property is significantly important when considering reduced density schemes to permit having a usable channel in extreme case scenarios.

\section{CONCLUSION}

In this paper, various Multi-Carrier transmission scenarios have been discussed in terms of pulse shape selection based on interference levels. Interference versus normalized Doppler spread for several delay profiles is provided in addition to the power received versus offset between the frequency-time index of the transmitter and the receiver. The interference localization properties of the pulse shapes and their importance are also analyzed. It was shown that for scenarios where Doppler spread is not the main concern, Rect pulses (corresponding to the conventional CP-OFDM) perform the best thanks to the $\mathrm{CP}$. However, if the delay spread went beyond the CP, Rect pulse loses its advantage. On the other hand, OBE pulses have the best performance when delay spread is not a major concern. Analysis was provided at the level of interference localization where neither delay nor Doppler spread is negligible. We suggest that in such extreme case scenarios, it is better to 
use pulses that have a higher level but localized interference like the Gauss and $\mathrm{RRC}^{1}$ pulses. This is due to the fact that the localization of their interference will be useful in density reduction. It is further interesting to check the impact of the interference localization properties on the channel estimation and interference cancellation techniques.

\section{REFERENCES}

[1] S. Weinstein and P. Ebert, "Data transmission by frequency-division multiplexing using the discrete fourier transform," IEEE transactions on Communication Technology, vol. 19, no. 5, pp. 628-634, 1971.

[2] C. Y. Wong, R. S. Cheng, K. B. Lataief, and R. D. Murch, "Multiuser OFDM with adaptive subcarrier, bit, and power allocation," IEEE Journal on selected areas in communications, vol. 17, no. 10, pp. 1747-1758, 1999.

[3] P. Schniter, "Low-complexity equalization of OFDM in doubly selective channels," IEEE Transactions on Signal processing, vol. 52, no. 4, pp. 1002-1011, 2004.

[4] C.-P. Li, J. Jiang, W. Chen, T. Ji, and J. Smee, "5g ultra-reliable and low-latency systems design," in 2017 European Conference on Networks and Communications (EuCNC). IEEE, 2017, pp. 1-5.

[5] L. Bernado, T. Zemen, F. Tufvesson, A. F. Molisch, and C. F. Mecklenbräuker, "Delay and doppler spreads of nonstationary vehicular channels for safety-relevant scenarios," IEEE Transactions on Vehicular Technology, vol. 63, no. 1, pp. 82-93, 2013.

[6] R. W. Chang, "Synthesis of band-limited orthogonal signals for multichannel data transmission," Bell System Technical Journal, vol. 45, no. 10, pp. 1775-1796, 1966

[7] C. Siclet and P. Siohan, "Design of BFDM/OQAM systems based on biorthogonal modulated filter banks," in Globecom'00-IEEE. Global Telecommunications Conference. Conference Record (Cat. No. 00CH37137), vol. 2. IEEE, 2000, pp. 701-705.

[8] R. H. Clarke, "A statistical theory of mobile-radio reception," Bell system technical journal, vol. 47, no. 6, pp. 957-1000, 1968.

[9] A. H. El Husseini, E. P. Simon, and L. Ros, "Second-order autoregressive model-based kalman filter for the estimation of a slow fading channel described by the Clarke model: Optimal tuning and interpretation," Digital Signal Processing, vol. 90, pp. 125-141, 2019.

[10] P. Bello, "Characterization of randomly time-variant linear channels," IEEE transactions on Communications Systems, vol. 11, no. 4, pp. 360393, 1963

[11] D. Pinchon and P. Siohan, "Closed-form expressions of optimal short PR FMT prototype filters," in 2011 IEEE Global Telecommunications Conference-GLOBECOM 2011. IEEE, 2011, pp. 1-5.

[12] S. Daumont, B. Rihawi, and Y. Lout, "Root-raised cosine filter influences on PAPR distribution of single carrier signals," in 2008 3rd International Symposium on Communications, Control and Signal Processing. IEEE, 2008, pp. 841-845.

[13] Mathworks. Propagation channel models. [Online]. Available: https //www.mathworks.com/help/lte/ug/propagation-channel-models.html

[14] A. Hamdan, L. Ros, H. Hijazi, C. Siclet, and A. Al-Ghouwayel, "On multi-carrier systems robustness to Doppler in fast varying flat fading wireless channel," Digital Signal Processing, vol. 117, p. 103189, 2021. [Online]. Available: https://hal.archives-ouvertes.fr/hal-03325410

\section{APPENDIX A}

DERIVATION OF THE EXPECTED RECEIVED POWER (12)

Using Eq. 10 and Eq. 11, the expected received power is:

$$
\begin{aligned}
\bar{P}_{m, m^{\prime}, n, n^{\prime}}= & \boldsymbol{E}\left[P\left(m, m^{\prime}, n, n^{\prime}\right)\right] \\
= & \sum_{q, q^{\prime}} \sum_{l, l^{\prime}} \tilde{g}^{*}\left[q-n^{\prime} N\right] \tilde{g}\left[q^{\prime}-n^{\prime} N\right] \\
& \times g[q-n N-l] g^{*}\left[q^{\prime}-n N-l^{\prime}\right] \\
& \times \boldsymbol{E}\left[h[q, l] h^{*}\left[q^{\prime}, l^{\prime}\right]\right] \\
& \times e^{-j 2 \pi \frac{(q-n N)\left(m^{\prime}-m\right)-m^{\prime} N\left(n^{\prime}-n\right)+l m}{M}} \\
& \times e^{j 2 \pi \frac{\left(q^{\prime}-n N\right)\left(m^{\prime}-m\right)-m^{\prime} N\left(n^{\prime}-n\right)+l^{\prime} m}{M}} \\
& \times \boldsymbol{E}\left[c_{m, n}\right],
\end{aligned}
$$

or equivalently:

$$
\begin{aligned}
\bar{P}_{m, m^{\prime}, n, n^{\prime}}= & \sum_{q, q^{\prime}} \sum_{l, l^{\prime}} \tilde{g}^{*}\left[q-n^{\prime} N\right] \tilde{g}\left[q^{\prime}-n^{\prime} N\right] \\
& \times g[q-n N-l] g^{*}\left[q^{\prime}-n N-l^{\prime}\right] \\
& \times e^{j 2 \pi \frac{\left(q^{\prime}-q\right)\left(m^{\prime}-m\right)+\left(l^{\prime}-l\right) m}{M}} \\
& \times R_{h}\left(q, q^{\prime}, l, l^{\prime}\right) \sigma_{c}^{2},
\end{aligned}
$$

where $R_{h}\left(q, q^{\prime}, l, l^{\prime}\right)$ is the multi-variable channel's auto-correlation function obtained by $R_{h}\left(q, q^{\prime}, l, l^{\prime}\right)=$ $\boldsymbol{E}\left[h[q, l] h^{*}\left[q^{\prime}, l^{\prime}\right]\right]$, and $\sigma_{c}^{2}$ is the transmitted symbol average power. Following the assumptions of unitary average transmission power $\left(\sigma_{c}^{2}=1\right)$, WSSUS channel [10] $\left(R_{h}\left(q, q^{\prime}, l, l^{\prime}\right)=0\right.$ for $\left.l \neq l^{\prime}\right)$, and Jakes Spectrum [8] Rayleigh channel $\left(R_{h}\left(q, q^{\prime}, l, l\right)=\sigma_{l}^{2} J_{0}\left(2 \pi F_{d} T_{S a}\left(q^{\prime}-q\right)\right)\right.$ where $J_{0}$ is the Bessel function of the first kind and order 0 and $\sigma_{l}^{2}$ the power/variance of the $l^{\text {th }}$ path), we obtain:

$$
\begin{aligned}
\bar{P}_{m, m^{\prime}, n, n^{\prime}}= & \sum_{q, q^{\prime}} \sum_{l} \tilde{g}^{*}\left[q-n^{\prime} N\right] \tilde{g}\left[q^{\prime}-n^{\prime} N\right] \\
& \times g[q-n N-l] g^{*}\left[q^{\prime}-n N-l\right] \\
& \times e^{j 2 \pi \frac{\left(q^{\prime}-q\right)\left(m^{\prime}-m\right)}{M}} \\
& \times \sigma_{l}^{2} J_{0}\left(2 \pi F_{d} T_{S a}\left(q^{\prime}-q\right)\right) .
\end{aligned}
$$

Replacing the variable $q$ and $q^{\prime}$ using $q=\gamma+n^{\prime} N$ and $q^{\prime}=\gamma^{\prime}+n^{\prime} N$ respectively, we get:

$$
\begin{aligned}
\bar{P}_{m, m^{\prime}, n, n^{\prime}} & =\sum_{\gamma, \gamma^{\prime}} \sum_{l} g\left[\gamma+N\left(n^{\prime}-n\right)-l\right] \\
& \times g^{*}\left[\gamma^{\prime}+N\left(n^{\prime}-n\right)-l\right] \\
& \times \tilde{g}^{*}[\gamma] \tilde{g}\left[\gamma^{\prime}\right] e^{j 2 \pi \frac{\left(m^{\prime}-m\right)\left(\gamma^{\prime}-\gamma\right)}{M}} \\
& \times \sigma_{l}^{2} J_{0}\left(2 \pi F_{d} T_{S a}\left(\gamma^{\prime}-\gamma\right)\right) .
\end{aligned}
$$

Introducing the index $\Delta=\left[\Delta_{S}, \Delta_{C}\right]=\left[n^{\prime}-n, m^{\prime}-m\right]$, we finally have:

$$
\begin{aligned}
\bar{P}_{\Delta}=\sum_{l} \sum_{\gamma} \sum_{\gamma^{\prime}} g\left[\gamma+N \Delta_{S}-l\right] g^{*}\left[\gamma^{\prime}+N \Delta_{S}-l\right] \\
\quad \times \tilde{g}^{*}[\gamma] \tilde{g}\left[\gamma^{\prime}\right] e^{j 2 \pi \frac{\Delta_{C}\left(\gamma^{\prime}-\gamma\right)}{M}} \\
\quad \times \sigma_{l}^{2} J_{0}\left(2 \pi F_{d} T_{S a}\left(\gamma^{\prime}-\gamma\right)\right),
\end{aligned}
$$

which corresponds to Eq. 12. 